\title{
ELEMENTARY DIVISOR THEOREM FOR NONCOMMUTATIVE PIDS
}

\author{
ROBERT M. GURALNICK, LAWRENCE S. LEVY AND CHARLES ODENTHAL
}

(Communicated by Donald S. Passman)

\begin{abstract}
We prove that, over a PID, if two matrices $\mathbf{A}$ and $\mathbf{B}$ have the same size, present isomorphic modules and have $\operatorname{rank} \geq 2$, then $\mathbf{A}$ is equivalent to $\mathbf{B}$. This answers a question raised by Nakayama in 1938. Our solution makes use of a number of facts about the algebraic $K$-theory of noetherian rings.
\end{abstract}

Let $\Lambda$ be a PID, that is, an integral domain (not necessarily commutative) in which every left ideal and every right ideal is principal. We call $m \times n$ matrices $\mathbf{A}$ and $\mathbf{B}$ over $\Lambda$ equivalent, and write $\mathbf{A} \sim \mathbf{B}$, if $\mathbf{B}=\mathbf{P A Q}$ for invertible matrices $\mathbf{P}$ and $\mathbf{Q}$ over $\Lambda$. It is well known [ $\left.\mathbf{T}{ }^{\prime} \mathbf{3 7}, \mathbf{A} \mathbf{~}^{\mathbf{3}} \mathbf{3 8}, \mathbf{J}{ }^{\prime} \mathbf{4 3}\right]$ that every such $\mathbf{A}$ is equivalent to a diagonal matrix $\mathbf{D}$ in which each diagonal entry is a total divisor of the next, that is,

$$
\mathbf{D}=\operatorname{diag}\left(d_{1}, d_{2}, \ldots\right) \quad \text { where each } \Lambda d_{i} \cap d_{i} \Lambda \supseteq \Lambda d_{i+1} \Lambda .
$$

Three obvious invariants for the equivalence class of $\mathbf{A}$ are $m, n$ and the isomorphism class of the left $\Lambda$-module

$$
U=\Lambda^{n} /\left(\Lambda^{m} \mathbf{A}\right) \cong \bigoplus_{i} \Lambda / \Lambda d_{i}
$$

presented by $\mathbf{A}$. Nakayama [ $\left.\mathbf{N}^{\prime} \mathbf{3 8}\right]$ refined this by observing that the isomorphism classes of the left $\Lambda$-modules $\Lambda / \Lambda d_{i}$ (each counted as often as it occurs) are invariants of the equivalence class of $\mathbf{A}$. However, the main purpose of his paper was to lament the fact that very little is known about the converse question: What invariants other than $m, n$ and the isomorphism class of $U$ are needed to determine the equivalence class of $\mathbf{A}$ ?

It was apparently well known that additional invariants are needed, even in the case of $1 \times 1$ matrices. For explicit examples, see [LR '74, 4.6, §5] and [GL '88]. The surprising answer to Nakayama's question is that, except in rank 1, there are no additional invariants:

ElEMENTARY DivisoR THEOREM. If $\operatorname{rank}(\mathbf{A}) \geq 2$, then $m, n$ and the isomorphism class of $U$ form a complete set of invariants for the equivalence class of A.

For completeness we note that the situation where $\operatorname{rank}(\mathbf{A})=1$ but $(m, n) \neq$ $(1,1)$ is essentially the same as the $1 \times 1$ case. Let $[a]$ and $[b]$ be inequivalent $1 \times 1$

Received by the editors July $21,1987$.

1980 Mathematics Subject Classification (1985 Revision). Primary 16A64, 15A33; Secondary $16 \mathrm{~A} 04$.

This research was partially supported by NSF grants DMS-8700961 and DMS-8720430.

(C) 1988 American Mathematical Society $0002-9939 / 88 \$ 1.00+\$ .25$ per page 
matrices such that $\Lambda / \Lambda a \cong \Lambda / \Lambda b$, and let $\mathbf{A}$ and $\mathbf{B}$ be $m \times n$ matrices with $a$ and $b$ respectively, in their $(1,1)$-position and zeros elsewhere. Then $\mathbf{A}$ and $\mathbf{B}$ are inequivalent but the $\Lambda$-module (0.2) presented by $\mathbf{A}$ is isomorphic to the analogous $\Lambda$-module presented by $\mathbf{B}$.

The Elementary Divisor Theorem above was proved in [GL '88] for the case that $\Lambda$ is module-finite over its center. In the present paper, we show how to extend that proof to the full noncommutative situation.

OUTLINE OF PROOF. It is convenient to take a more abstract point-of-view, replacing matrices with module homomorphisms. We call $\Lambda$-module homomorphisms $f, g: M \rightarrow N$ (often acting on the right) equivalent, and write $f \sim g$, if $g=\varphi f \vartheta$ for automorphisms $\varphi$ and $\vartheta$ of $M$ and $N$ respectively. Letting $\alpha$ denote right multiplication by the $m \times n$ matrix $\mathbf{A}$ yields a free resolution

$$
\Lambda^{m} \stackrel{\alpha}{\rightarrow} \Lambda^{n} \stackrel{f}{\rightarrow} U
$$

where $U$ is the module in (0.2). Similarly, right multiplication by $\mathbf{B}$ yields another resolution $(\beta, g)$ of $U$, since we are assuming that $\mathbf{A}$ and $\mathbf{B}$ present isomorphic modules.

We want to prove that $\alpha \sim \beta$.

The problem is easily reduced to the case that $\alpha$ is one-to-one, as in the proof of [GL '88, 3.6]. (The kernel of $\alpha$ splits off since $\Lambda$ is a PID.) Thus, from now on we will be concerned with a presentation

$$
K \stackrel{\alpha}{\mapsto} P=\Lambda^{n} \stackrel{f}{\rightarrow} U
$$

of a left $\Lambda$-module $U$, where $\alpha$ denotes the inclusion map. What we want to prove is that $U$ is uniquely presentable by $P$, that is, if $g: P \rightarrow U$ is any other presentation of $U$ by $P$, then $g \sim f$.

It is easy to reduce the problem to the case that $U$ has finite length, as in the proof of [GL '88, 3.5] (because the torsion submodule of $U$ splits off).

The first part of what we prove in this paper is that the theorem is "stably" true, that is, there is an $s$ such that $U^{s}$ is uniquely presentable by $P^{s}$. This is done in $\S 1$.

It follows that $f^{s} \sim\left(g \oplus f^{s-1}\right)$ since both are presentations of $U^{s}$ by $P^{s}$. The final step is then to show that, when the free module $P$ has rank $\geq 2, f^{s-1}$ can be cancelled, yielding $f \sim g$. This is done in $\S \S 2$ and 3 .

There are two difficulties in this proof that are not present when $\Lambda$ is modulefinite over its center. First is the presence of completely faithful $\Lambda$-modules of finite length, that is, faithful modules $U$ of finite length such that every nonzero submodule of every homomorphic image of $U$ is faithful. We reduce the cancellation problem to the case of presentations of unfaithful modules in $\S 2$, and then do the unfaithful case in $\S 3$. It is in this last part that the ideas from $K$-theory are used.

The second difficulty is that localization at maximal ideals of the center of $\Lambda$ (one of the main tools in [GL '88]) does not seem very useful in the present situation, where $\Lambda$ may not be module-finite over its center and we do not know very much about the center itself. Fortunately, we are able to avoid localization, in the present paper. 
For the remainder of this paper, $\Lambda$ denotes a PID, and "module" means "finitely generated module" unless otherwise stated. We want to show that every left $\Lambda$ module $U$ of finite length is uniquely presentable by every free module $P$ by which it can be presented, as in (0.4).

1. Stable unique presentability. We begin by quoting [LR $\mathbf{7 4}, 1.5]$.

1.1 Lifting AND StRAightening TheOREM. Consider a surjection of modules over any ring

$$
f: P=P_{1} \oplus P_{2} \oplus \cdots \oplus P_{n} \rightarrow U=U_{1} \oplus U_{2} \oplus \cdots \oplus U_{n}
$$

where $P$ is projective and $U / \operatorname{rad} U$ is semisimple (e.g. any module $U$ of finite length). Suppose that

$$
\text { For each } i \text { there exist surjections: } P_{i} \rightarrow U_{i} \text { and } P_{n} \rightarrow U_{i} .
$$

Then the $P_{i}$ can be isomorphically replaced in (1.1.1) to achieve $f\left(P_{i}\right)=U_{i}$ for every $i$ ("lifting"). Moreover, this replacement can be done in such a way that the first $n-1$ restricted maps $f: P_{i} \rightarrow U_{i}$ become equivalent to $n-1$ arbitrarily selected surjections: $P_{i} \rightarrow U_{i}$ ("straightening").

The phrase "isomorphically replaced", in the theorem, means that the new $P_{i}$ is isomorphic to the old one. An immediate consequence of the theorem is

1.2 Corollary. Suppose that $U_{n}$ is uniquely presentable by $P_{n}$. Then $U$ is uniquely presentable by $P$.

One situation in which unique presentability is easily shown to hold is given in [LR '74, 1.9].

1.3 LEMMA. Let $T$ be a 2 -sided ideal of any ring $R$, and let $H$ be any noetherian $R$-module. Then any two surjections: $H \rightarrow H / T H$ are equivalent.

We now return to our PID $\Lambda$.

1.4 STABLE INVARIANT FACTOR THEOREM. Let $K$ be a submodule of a free $\Lambda$-module $P$. Suppose that $P / K$ is unfaithful of finite length. Then for some $s$ there exist compatible decompositions

$$
\begin{gathered}
P^{s}=P_{1} \oplus \cdots \oplus P_{n} \text { and } \\
K^{s}=T_{1} P_{1} \oplus \cdots \oplus T_{n} P_{n} \quad\left(T_{1} \subseteq \cdots \subseteq T_{n}\right)
\end{gathered}
$$

with each $T_{i}$ a 2 -sided ideal of $\Lambda$ and each $P_{i} \cong \Lambda$.

PROOF. The proof is the same as the proof of [GL '88, 3.3], with the following minor changes. Since $\Lambda$ is a PID, all projective $\Lambda$-modules are free, and their uniform rank equals their rank as a free module. Moreover, the "genus" of a projective module merely becomes its isomorphism class. In a number of places, the preliminary results [GL '88, 3.1,3.2] refer to a regular element $d$ of a central subring $R$ such that $d U=0$. In all such cases, it suffices to take $d$ to be a regular element of $R$; and this always exists, by Goldie's theorem, because the annihilator of the unfaithful module $U$ is a 2 -sided ideal of the prime ring $\Lambda$, hence is essential as a left ideal.

We now obtain our desired stable unique presentability result. 
1.5 THEOREM. Let $f: P \rightarrow U$ be a presentation of a $\Lambda$-module of finite length. Then, for some $s, U^{s}$ is uniquely presentable by $P^{s}$.

PrOOF. We seek an integer $s$ for which there exist decompositions $P^{s}=\bigoplus_{i=1}^{n} P_{i}$ and $U^{s}=\bigoplus_{i=1}^{n} U_{i}$ with each $P_{i} \cong \Lambda$, each $U_{i}$ cyclic, and $U_{n} \cong \Lambda / T$ for some 2sided ideal $T$ of $\Lambda$. For then $U_{n}$ is uniquely presentable by $P_{n}$ (Lemma 1.3), and hence $U$ is uniquely presentable by $P$ (Corollary 1.2).

Let $K=\operatorname{ker}(f)$. It suffices to find an integer $s$ and decompositions

$$
P^{s}=\bigoplus_{i=1}^{n} \Lambda p_{i} \quad \text { and } \quad K^{s}=\bigoplus_{i=1}^{n} \Lambda d_{i} p_{i}
$$

such that $T=\Lambda d_{n}$ is a 2-sided ideal, for then $U=\bigoplus_{i} f\left(\Lambda p_{i}\right)$ with $f\left(\Lambda p_{n}\right) \cong \Lambda / T$.

We can suppose that $P$ has rank $r \geq 2$, after replacing $f$ by $f^{2}$, if necessary. Let $K=\operatorname{ker}(f)$. Applying the diagonalization theorem mentioned in $(0.1)$ to the matrix of the inclusion map: $K \subseteq P$, we get a pair of decompositions

$$
P=\bigoplus_{i=1}^{r} \Lambda p_{i} \quad \text { and } \quad K=\bigoplus_{i=1}^{r} \Lambda d_{i} p_{i}
$$

where each $d_{i}$ is a total divisor of $d_{i-1}(i>1)$. Since $U$ has finite length and $P$ $\operatorname{rank}(P)=r$, every $d_{i}$ and $p_{i}$ is nonzero.

Let $P^{\prime}=\bigoplus_{i \neq 1} \Lambda p_{i}$ and $K^{\prime}=\bigoplus_{i \neq 1} \Lambda d_{i} p_{i}$. Since every $d_{i}$ is a total divisor of $d_{i-1}$ we have $\Lambda d_{1} \Lambda \subseteq \Lambda d_{i}$ for all $i>1$. Therefore $P^{\prime} / K^{\prime}$ is annihilated by $d_{1}$. Let $s$ be the integer obtained by applying the Stable Invariant Factor Theorem to the unfaithful module $P^{\prime} / K^{\prime}$.

Since $P=\Lambda p_{1} \oplus P^{\prime}$ and $K=\Lambda d_{i} p_{i} \oplus K^{\prime}$, the decompositions of $\left(P^{\prime}\right)^{s}$ and $\left(K^{\prime}\right)^{s}$ provided by the Stable Invariant Factor Theorem yield the decomposition needed in (1.5.1).

\section{Reduction to unfaithful modules.}

2.1 THEOREM. Suppose that every unfaithful $\Lambda$-module $U$ of finite length is uniquely presentable by every free module of rank $\geq 2$ that can be mapped onto $U$. Then the same conclusion holds if $U$ is faithful (and of finite length).

ProOF. Let $f: P \rightarrow U$ be a presentation with $\operatorname{ker}(f)=K$, where $U$ is not necessarily faithful. Choose a pair of decompositions, as in (1.5.2) with each $d_{i}$ a total divisor of $d_{i-1}(i>1)$. This gives a pair of decompositions

$$
P=\bigoplus_{i=1}^{n} P_{i} \quad \text { and } \quad U=\bigoplus_{i=1}^{n} U_{i}
$$

with each $P_{i} \cong \Lambda$ and each $f\left(P_{i}\right)=U_{i}$. Moreover, since each $d_{i}$ is a total divisor of $d_{i-1}$ the module $U^{\prime}=\bigoplus_{i=2}^{n} U_{i}$ is annihilated by $d_{1}$, hence is unfaithful.

Now suppose that $P$ has rank $\geq 3$. Then, by hypothesis, $U^{\prime}$ is uniquely presentable by $P^{\prime}=\bigoplus_{i=2}^{n} P_{i}$. Hence applying Corollary 1.2 to the presentation

$$
f: P=P_{1} \oplus P^{\prime} \rightarrow U=U_{1} \oplus U^{\prime},
$$

shows that $U$ is uniquely presented by $P$, as desired.

Unfortunately, this simple argument fails when $n=2$ because $P^{\prime}$ then has rank 1 . The only way out seems to be to modify the proof of the Lifting and Straightening 
Theorem, which we do in the next lemma. To apply the lemma, recall that every $\Lambda$-module of finite length is the direct sum of a completely faithful module and an unfaithful module [ER '70, 3.9].

2.2 LEMMA. Let $f: P \rightarrow U=V \oplus W$ be a presentation of a $\Lambda$-module, with $P$ free of rank 2, $U$ of finite length, $V$ completely faithful, and $W$ unfaithful. Suppose that $W$ is uniquely presentable by $P$. Then $U$ is uniquely presentable by $P$.

ProOF. Let $P=\Lambda p_{1} \oplus \Lambda p_{2}$. Since $P$ can be mapped onto $W$, the diagonalization argument in (1.5.2) gives a decomposition $W=\Lambda w_{1} \oplus \Lambda w_{2}$.

Recall from [LR '74, Lemma 1.11] that, over any ring, if $S$ is a projective module not of finite length and $S$ can be mapped onto a module $L$ of finite length, and $C$ is any completely faithful module of finite length, then $S$ can be mapped onto $C \oplus L$.

Applying this with $S=\Lambda$ and $L=0$, we see that $\Lambda$ can be mapped onto $V$, say $V=\Lambda v$. A second application of this lemma shows that $\Lambda$ can be mapped onto $\Lambda v \oplus \Lambda w_{1}$. Applying the Lifting and Straightening Theorem to the presentation

$$
f: P=\Lambda p_{1} \oplus \Lambda p_{2} \rightarrow U=\left(\Lambda v \oplus \Lambda w_{1}\right) \oplus \Lambda w_{2}
$$

we get a new decomposition $P=\Lambda p_{1} \oplus \Lambda p_{2}$ such that $f\left(\Lambda p_{1}\right)=\Lambda v \oplus \Lambda w_{1}$ and $f\left(\Lambda p_{2}\right)=\Lambda w_{2}$. After a change of notation we get

$$
f\left(p_{1}\right)=v+w_{1} \quad \text { and } \quad f\left(p_{2}\right)=w_{2} .
$$

Let $\pi_{v}$ and $\pi_{W}$ be the projection maps from $P$ to $\Lambda v$ and $W$ respectively.

Now consider another presentation $g: S \rightarrow X$ with $S \cong P$ and $X \cong U$. We show that $f \sim g$ by obtaining decompositions $f \sim f_{1} \oplus f_{2}$ and $g \sim g_{1} \oplus g_{2}$ in which each $f_{i} \sim g_{i}$.

Since $X \cong U$, there is a decomposition $X=\Lambda y \oplus Z$ with $\Lambda y \cong \Lambda v$ and $Z \cong W$. Since we are assuming that $W$ is uniquely presentable by $P$, we have

$$
\left(\pi_{W} f: P \rightarrow W\right) \sim\left(\pi_{Z} g: S \rightarrow Z\right) .
$$

This yields decompositions $S=\Lambda s_{1} \oplus \Lambda s_{2}$ and $Z=\Lambda z_{1} \oplus \Lambda z_{2}$ such that

$$
\left(\pi_{W} f: \Lambda p_{i} \rightarrow \Lambda w_{i}\right) \sim\left(\pi_{Z} g: \Lambda s_{i} \rightarrow \Lambda z_{i}\right) \quad(i=1,2)
$$

where each $s_{i} \rightarrow z_{i}$ and

$$
\operatorname{ann}\left(w_{1}\right)=\operatorname{ann}\left(z_{1}\right)=(\text { say }) K .
$$

So we have $\operatorname{ker}\left(\pi_{W} f \mid \Lambda p_{1}\right)=K p_{1}$ and $\operatorname{ker}\left(\pi_{Z} g \mid \Lambda s_{1}\right)=K s_{1}$.

Now we make a sequence of basis changes in $S$. The basis changes will be elementary, replacing $s_{1}$ by an expression of the form $s_{1}+t s_{2}$ or replacing $s_{2}$ by $s_{2}+t s_{1}$ where $t$ is an element of the annihilator $T$ of $Z$. Taking $t \in T$ guarantees that (2.2.4) still holds after the old $s_{i}$ is replaced by the new $s_{i}$, because $\pi_{Z} g\left(s_{i}\right)$ remains unchanged.

We have $T \neq 0$ since $Z$ is unfaithful. The following fact will be used repeatedly.

$$
T y^{\prime}=\Lambda y^{\prime} \quad\left(\forall y^{\prime} \in \Lambda y\right) .
$$

Clearly $T y^{\prime} \subseteq \Lambda y^{\prime}$. If equality did not hold, then the nonzero module $\Lambda y^{\prime} / T y^{\prime}$ would be annihilated by the nonzero ideal $T$, contrary to complete faithfulness of $\Lambda y$. 
Our first claim is that there is a basis change $s_{2} \rightarrow s_{2}+t s_{1}$ that achieves $\pi_{y} g\left(\Lambda s_{2}\right)=\Lambda y$. Applying (3) in the proof of the Building Lemma [LR, 1.3] to the surjection $\pi_{y} g: \Lambda s_{2} \oplus \Lambda s_{1} \rightarrow \Lambda y$ gives a homomorphism $\vartheta: \Lambda s_{2} \rightarrow \Lambda s_{1}$ such that $\pi_{y} g(1+\vartheta)\left(\Lambda s_{2}\right)=\Lambda y$. We have $\vartheta\left(s_{2}\right)=t s_{1}$ for some $t \in \Lambda$. We then have $\Lambda y=\Lambda \pi_{y} g\left(s_{2}+t s_{1}\right)$ as needed. Moreover, $t \pi_{y} g\left(s_{1}\right) \in \Lambda y$ so, by (2.2.6), we can take $t \in T$ as claimed.

Since $\pi_{y} g\left(\Lambda s_{2}\right)=\Lambda y$ we can replace $y$ by a different element of $\Lambda y$ to achieve $\pi_{y} g\left(s_{2}\right)=y$.

Next we claim that there is a basis change $s_{1} \rightarrow s_{1}+t s_{2}$ that achieves $\pi_{y} g\left(s_{1}\right)=$ 0 . For some $t \in \Lambda$ we have $\pi_{y} g\left(s_{1}\right)=-t y=-t \pi_{y} g\left(s_{2}\right)$ as needed. Again, by (2.2.6), we can take $t \in T$.

Now that we have "erased" the unknown image of $\Lambda s_{1}$ in $\Lambda y$, we rebuild this image to suit our needs.

Since $\Lambda v \cong \Lambda y$, there is an element $t \in \Lambda$ such that $\Lambda t y=\Lambda y$ and $\operatorname{ann}(t y)=\operatorname{ann}(v)$. Again we can take $t \in T$. After replacing $s_{1}$ by $s_{1}+t s_{2}$ we have $\pi_{y} g\left(\Lambda s_{1}\right)=\Lambda t y=$ $\Lambda y$.

After replacing $y$ by $t y$ we have $\pi_{y} g\left(s_{1}\right)=y$ and $\operatorname{ann}(y)=\operatorname{ann}(v)=($ say) $H$. Therefore, by (2.2.5),

$$
\operatorname{ann}\left(y+z_{1}\right)=\operatorname{ann}(y) \cap \operatorname{ann}\left(z_{1}\right)=H \cap K=\operatorname{ann}\left(v+w_{1}\right) .
$$

Since $g\left(s_{1}\right)=y+z_{1}$ we now have $\operatorname{ker}\left(g \mid \Lambda s_{1}\right)=(H \cap K) s_{1}$ and $\operatorname{ker}\left(f \mid \Lambda p_{1}\right)=$ $(H \cap K) p_{1}$. Therefore

$$
\left(g \mid \Lambda s_{1}\right) \sim\left(f \mid \Lambda p_{1}\right) .
$$

We no longer need the image of $\Lambda s_{2}$ in $\Lambda y$. So, by means of one more basis change of the form $s_{2} \rightarrow s_{2}+t s_{1}$ we erase it, replacing it with 0 .

Setting $i=2$ in (2.2.4), and observing that the projection maps $\pi$ now have no effect, we get $\left(g \mid \Lambda s_{2}\right) \sim\left(f \mid \Lambda p_{2}\right)$ which, together with (2.2.7), completes the proof of the lemma.

\section{Unfaithful case.}

3.1 Notation. Let $f, g: P=\Lambda^{n} \rightarrow U$ be presentations of an unfaithful left $\Lambda$ module of finite length, with $\operatorname{ker}(f)=K$ and $\operatorname{ker}(g)=L$. We have $T U=0$ for some nonzero 2-sided ideal $T$ of $\Lambda$. Let $E(P)$ denote the endomorphism ring of $P$. We define

$$
\operatorname{hom}(f, g)=\{\varphi \in E(P) \mid K \varphi \subseteq L\}=\{\varphi \in E(P) \mid(\exists \vartheta \in E(U)) \varphi g=f \vartheta\} \text {. }
$$

In particular, we let hom $(f, f)=E(f)=E$. Note that hom $(f, g)$ is a left $E$-module.

Let $\Gamma=\Lambda_{n \times n}$. We write elements of $P$ as rows. So every element of $E(P)$ becomes right multiplication by a unique element of $\Gamma$, and we make the identification $E(P)=\Gamma$. Thus $E=E(f)$ is a subring of $\Gamma$. Let $\mathscr{T}=T_{n \times n}$. Since $T U=0$, we have

$$
\mathscr{T} \subseteq H \text { and } \mathscr{T} \subseteq E
$$

In fact, $\mathscr{T}$ is a 2-sided ideal of both $E$ and $\Gamma$.

For any $\lambda$-module homomorphism $h: S \rightarrow V$ we define the homomorphism $f \oplus$ $h: P \oplus S \rightarrow U \oplus V$ by $(p, s) \rightarrow(p f, s h)$. It therefore makes sense to speak of the category $\operatorname{div}(f)$ of all direct summands of the maps $f^{n}(n=1,2, \ldots)$. 
3.2 DRESS'S LEMMA. The functor hom $(f, \ldots)$ is a category equivalence between the $\operatorname{div}(f)$ and $\operatorname{div}(E)=\{$ finitely generated projective left $E$-modules $\}$. In particular, $g \sim f$ if and only if hom $(f, g) \cong E$ as left $E$-modules.

PROOF. Dress's well-known observation [D '69, p. 985] is that, if $F$ is a left module over a ring $R$, then the functor $\operatorname{hom}(F, \ldots)$ is a category equivalence between $\operatorname{div}(F)$ and $\operatorname{div}(E(F))$, the inverse functor being $F \otimes_{E(F)}(\cdots)$.

Let $T_{2}(\Lambda)$ be the ring of $2 \times 2$ upper triangular matrices over $\Lambda$. In [GL '88, 1.8] a functor $f \rightarrow M(f)$ is described that is a category equivalence between the category of homomorphisms of left $\Lambda$-modules and a subcategory of the category of left $T_{2}(\Lambda)$-modules. (Actually, in that discussion, homomorphisms act on the left, and lower triangular matrices are used.)

To obtain our lemma from Dress's original version, let $F=M(f)$ and compose the functor $M(\cdots)$ in the previous paragraph with Dress's functor.

\subsection{LEMMA. E/T is a left and right artinian ring.}

ProOF. Note that (by considering Morita equivalence) a ring $R$ is artinian if and only if, for some $s$, the matrix ring $R_{s \times s}$ is artinian.

So it suffices to prove the lemma with $E\left(f^{s}\right)$ in place of $E(f)$. Choose $s$ such that the Stable Invariant Functor Theorem 1.4 holds for $K \subseteq P$. After the change of notation that replaces $f$ by $f^{s}$, the decomposition given by the Stable Invariant Factor Theorem takes the form $P=\Lambda^{n}$ and $K=\bigoplus_{i=1}^{n} T_{i}$ where each $T_{i}$ is a 2-sided ideal of $\Lambda$ containing $T$.

The subring $E$ of $\Lambda_{n \times n}$ consists of all matrices $\varphi$ such that, for all $(i, j)$, we have $T_{i} \varphi_{i j} \subseteq T_{j}$. Since each $T_{i}$ is a 2 -sided ideal, each subset $E_{i j}$ of $E$ is therefore a 2-sided ideal of $\Lambda$ containing $T$, and $E=\sum_{i j} E_{i j}$.

It follows that $E$ is a finitely generated $\Lambda$-module on the left and on the right. Hence $E / \mathscr{T}$ is a finitely generated left and right module over the artinian ring $\Lambda / T$. In particular, $E / \mathscr{T}$ is an artinian ring.

3.4 THEOREM. Any two presentations $f, g: P=\Lambda^{n} \rightarrow U$, where $U$ is unfaithful of finite length, and $n \geq 2$, are equivalent.

PROOF. By Theorem 1.5, $U^{s}$ is uniquely presentable by $P^{s}$ for some $s$. Therefore $f^{s} \sim g \oplus f^{s-1}$. In particular, $g \in \operatorname{div}(f)$. Applying the functor in Dress's Lemma, we get an isomorphism of projective left $E$-modules:

$$
E^{s} \cong H \oplus E^{s-1} \quad \text { where } H=\operatorname{hom}(f, g) \text {. }
$$

To complete the proof of the theorem, it now suffices to show $E \cong H$.

By (3.1.2) we can set $\bar{H}=H / \mathscr{T}$ and $\bar{E}=E / \mathscr{T}$. We also set $\bar{\Gamma}=\Gamma / \mathscr{T}$.

Let $\bar{\Lambda}=\Lambda / T$. Since free modules are projective, there is a natural identification $\bar{H}=\operatorname{hom}(\bar{f}, \bar{g})$ where $\bar{f}, \bar{g}: \bar{P}=\bar{\Lambda}^{n} \rightarrow U$ are the presentations of the $\bar{\Lambda}$-module $U$ induced by $f$ and $g$. Applying this to the situation $f=g$, we see that we can also make the identification $\bar{E}=E(\bar{f})$. Since the ring $\bar{\Lambda}$ is a homomorphic image of a $\mathrm{PID}, \bar{\Lambda}$ is artinian, and therefore has 1 in its stable range. Over a ring with 1 in its stable range, every module is uniquely presentable by every free module that presents it, by [W' $\mathbf{7 8}$, Theorem 4; or $\mathbf{G}$ ' $\left.^{\mathbf{8 2}}, 3.1\right]$. So $\bar{f} \sim \bar{g}$. Hence $\bar{E} \cong \bar{H}$ as left $\bar{E}$-modules. In particular, $\bar{H}=\bar{E} \bar{\alpha}$ for some $\bar{\alpha} \in \bar{H}$. Since $\bar{f} \sim \bar{g}$, any such $\bar{\alpha}$ is an 
element of $E(\bar{P})^{*}=\bar{\Gamma}^{*}$, where * denotes "units of". We claim

$$
\Gamma=\Gamma H=\Gamma E \text {. }
$$

Since $\bar{H}=\bar{E} \bar{\alpha}$ we have $H=\mathscr{T}+E \alpha$. So $\bar{\Gamma} \bar{H}=\bar{\Gamma} \bar{\alpha}=\left(\right.$ since $\left.\bar{\alpha} \in \bar{\Gamma}^{*}\right) \bar{\Gamma}$. Since both $\Gamma$ and $\Gamma H$ contain $\mathscr{T}$, the first equality in (3.4.2) follows. The second equality follows from the first one by setting $f=g$.

Conversely, let $\bar{\alpha} \in \bar{H} \cap \bar{\Gamma}^{*}$. Then $\bar{H}=\bar{E} \bar{\alpha}$. We claim

$$
H \cong E \Leftrightarrow \bar{\alpha} \in \bar{E}^{*} \Gamma^{*} \quad\left(\subseteq \bar{\Gamma}^{*}\right) .
$$

The product $\bar{E}^{*} \Gamma^{*}$ makes sense if we reduce the factor in $\Gamma$ modulo the 2-sided ideal $\mathscr{T}$ before multiplying.

Suppose $H \cong E$. Then $H=E \beta$ for some $\beta \in E$. We have $\beta \in \Gamma^{*}$ by (3.4.2). Then $\bar{H}=\bar{E} \beta=\bar{E} \bar{\alpha}$ shows $\bar{\alpha} \beta^{-1} \in \bar{E}^{*}$ as desired. Conversely, suppose $\bar{\alpha}=\bar{\varphi} \beta$ with $\bar{\varphi} \in \bar{E}^{*}$ and $\beta \in \Gamma^{*}$. Then $\bar{H}=\bar{E} \bar{\alpha}=\bar{E} \beta$ so $H=E \beta$. (Note that both $H$ and $E$ contain $\mathscr{T}$, and since $\beta \in \Gamma^{*}$, so does $E \beta$.) Hence $H=E \beta \cong E$.

Now we prove that $H \cong E$ by verifying the right-hand side of (3.4.3).

Let $\alpha \in \bar{H} \cap \bar{\Gamma}^{*}$ so $\bar{H}=\bar{E} \bar{\alpha}$. Then $A=(\bar{\alpha}, 1,1, \ldots, 1)$ is an isomorphism: $\bar{E}^{s} \cong \bar{H} \oplus \bar{E}^{s-1}$. In view of (3.4.1) we can apply the version of (3.4.3) that applies to the presentation $f^{s}: P^{s} \rightarrow U^{s}$, getting

$$
A=(\bar{\alpha}, 1,1, \ldots, 1) \in G L_{s}(\bar{E}) G L_{s}(\Gamma), \quad \text { say } A=x y .
$$

In what follows, we repeatedly use the following two facts about $\mathbf{K}_{1}$. If a $\operatorname{ring} R$ has $m$ in its stable range, then the natural map: $G L_{m}(R) \rightarrow \mathbf{K}_{1}(R)$ is a surjection, and the kernel of the map: $G L_{m+1}(R) \rightarrow \mathbf{K}_{1}(R)$ is the subgroup generated by elementary matrices.

Let $\nu_{\Lambda}$ denote "natural image in $\mathbf{K}_{1}(\Lambda)$ ". The PID $\Lambda$ has Krull dimension 1, therefore, by $\left[\mathbf{S}\right.$ '77, 2.1] has 2 in its stable range. So $\nu_{\Lambda}(y)=\nu_{\Lambda}(\gamma)$ for some $\gamma \in G L_{2}(\Lambda)$. Since $P$ has $\operatorname{rank} \geq 2$, we have $G L_{2}(\Lambda) \subseteq \Gamma^{*}$, so $\gamma \in \Gamma^{*}$. Let $\nu^{\prime}$ denote "natural image in $\mathbf{K}_{1}(\bar{E})$ ". Since the artinian ring $\bar{E}$ has 1 in its stable range, we have $\nu^{\prime}(x)=\nu^{\prime}(\bar{\varphi})$ for some $\bar{\varphi} \in \bar{E}^{*}$. Letting $\nu$ denote "natural image in $\mathbf{K}_{1}(\bar{\Gamma})$ ", we get $\nu(\bar{\alpha})=\nu(\bar{\varphi}) \nu(\gamma)=\nu(\bar{\varphi} \gamma)$ where we compute $\bar{\varphi} \gamma$ by viewing $\gamma$ as a matrix over $\Lambda$, and reducing its entries modulo $T$.

Since 1 is in the stable range of $\bar{\Gamma}$, and $\bar{\Gamma}$ consists of matrices of size at least $2 \times 2$ over $\bar{\Lambda}$, we now have $\bar{\alpha}=\bar{\varphi} \gamma \bar{\varepsilon}$ where $\bar{\varepsilon}$ is a product of elementary matrices over $\bar{\Lambda}$, hence can be lifted to a unit $\varepsilon \in \Gamma^{*}$. The relation $\bar{\alpha}=\bar{\varphi} \cdot(\gamma \varepsilon)$ now shows, by (3.4.3), that $H \cong E$.

\section{REFERENCES}

[A '38] K. Asano, Nichtcommutative Hauptidealringe, Actualités. Sci. Indust., no. 696, Hermann, Paris, 1938.

[D '69] A. Dress, On the decomposition of modules, Bull. Amer. Math. Soc. 75 (1969), 984-986.

[ER '70] D. Eisenbud and J. C. Robson, Modules over Dedekind prime rings, J. Algebra 16 (1970), 67-85.

[G '82] R. M. Guralnick, Matrix equivalence and isomorphism of modules, Linear Algebra Appl. 43 (1982), 125-136.

[GL '88] R. M. Guralnick and L. S. Levy, Presentations of modules when ideals need not be principal, Illinois J. Math. (to appear). 
[J '43] N. Jacobson, The theory of rings, Amer. Math. Soc. Colloq. Publ., vol. 37, Amer. Math. Soc., Providence, R.I., 1943.

[LR '74] L. S. Levy and J. C. Robson, Matrices and pair of modules, J. Algebra 29 (1974), 427-454. [N '38] T. Nakayama, A note on the elementary divisor theory in non-commutative domains, Bull. Amer. Math. Soc. 44 (1938), 719-723.

[S '77] J. T. Stafford, Stable structure of noncommutative noetherian rings, J. Algeb:a 47 (1977), 244-267.

[T '37] O. Teichmüller, Der Elementarteilersatz für nichtkommutative Ringe, S.-B.-Preuss Akad. Wiss., 1937.

[W '78] R. B Warfield, Jr., Stable equivalence of matrices and resolutions, Comm. Algebra 6 (1978), 1811-1828.

Department of Mathematics, University of Southern California, Los AnGELES, CALIFORNIA 90089-1113

Department of MATHEMATICS, UNIVERSity OF Wisconsin, MADison, Wisconsin 53706 